Revista Brasileira de

Engenharia Agrícola e Ambiental

v.16, n.11, p.1159-1165, 2012

Campina Grande, PB, UAEA/UFCG - http://www.agriambi.com.br

agriambi Protocolo 125.11 - 15/06/2011 • Aprovado em 20/07/2012

\title{
Diagnose nutricional com o uso de sensor óptico ativo em algodoeiro
}

\author{
Anamari V. de A. Motomiya ${ }^{1}$, José P. Molin ${ }^{2}$, \\ Wagner R. Motomiya ${ }^{3} \&$ G uilherme A. Biscaro ${ }^{4}$
}

\begin{abstract}
RESU M 0
Este trabalho objetivou avaliar a resposta espectral do dossel de plantas à variação de doses de nitrogênio (N) e sua relação com os teores foliares de N, índice de clorofila e produtividade na cultura do algodoeiro. 0 experimento foi conduzido em Chapadão do Sul, MS, no delineamento em blocos casualizados, com quatro repetições. Os tratamentos consistiram de cinco doses de $\mathrm{N}: 0,30,70,110$ e $150 \mathrm{~kg} \mathrm{ha}^{-1}$, divididas em duas aplicações aos 28 e 41 dias após a emergência utilizando-se, como fonte, o fertilizante ureia. As maiores correlações dos índices de clorofila e do índice de vegetação por diferença normalizada com a produtividade foram observadas na quarta leitura, aos 56 dias após a emergência, indicando que neste período a produtividade já pode estar comprometida caso haja falhas no suprimento de $\mathrm{N}$ à cultura. $0 \mathrm{~s}$ resultados obtidos indicaram que o sensor se torna mais sensível à variação do teor foliar de $\mathrm{N}$ conforme a planta se desenvolve mas não quando ela atinge um alto índice de área foliar e começa a entrar em senescência. Concluiu-se, então, que o sensoriamento remoto ao nível terrestre permite estimar indiretamente a quantidade de $\mathrm{N}$ absorvido, o índice de clorofila e a produtividade do algodoeiro.
\end{abstract}

Palavras-chave: adubação, Gossypium hirsutum, sensoriamento remoto

\section{Nutritional diagnosis with the use of active optical sensor in cotton}

\begin{abstract}
A B ST RAC T
This research aimed to evaluate the spectral response to variation of nitrogen levels and its relationship with leaf nitrogen, chlorophyll and yield in cotton crop. The experiment was conducted in Chapadão do Céu, M S, in a randomized block design with four replications. The treatments consisted of five $\mathrm{N}$ rates of $0,30,70,110$ and $150 \mathrm{~kg} \mathrm{ha}^{-1}$, divided in two applications at 28 and 41 days after emergence, using urea fertilizer as a source. The highest correlations of the chlorophyll index and normalized difference vegetation index with yield were observed in the fourth observation, at 56 days after emergence, indicating that in this period, yield may already be compromised if there is shortage in the supply of $\mathrm{N}$ to the crop. The results obtained indicated that the sensor becomes more sensitive to variations of nitrogen levels as the crop grows, but not when it reaches a high leaf area index and in the begining of the senescence. It is concluded that ground level remote sensing permits to estimate indirectly the amount of $\mathrm{N}$ absorbed, chlorophyll index and cotton yield.
\end{abstract}

Key words: fertilization, Gossypium hirsutum, remote sensing

\footnotetext{
${ }^{1}$ FCA/UFGD. C.P. 553, CEP 79804-970, Dourados, MS. Fone: (67) 3410-2415. E-mail: anamarimotomiya@ufgd.edu.br 2 ESALQ/U SP. C.P. 9, CEP 13418-900, Piracicaba, SP. Fone: (19) 3447-8502. E-mail: jpmolin@usp.br

3 Programa de Pós-Graduação em Genética e Melhoramento/U EM, Av. Colombo, 5790. CEP 87020-900, Maringá, PR. Fone: (44) $3011-8984$. E-mail: wagner.r.motomiya@monsanto.com

${ }^{4}$ FCA/U FGD. Fone: (67) 3410-2420. E-mail: guilhermebiscaro@ufgd.edu.br
} 


\section{INTRODUÇÃO}

A aplicação eficiente de nitrogênio (N) é importante para a obtenção de alta produtividade das culturas. Normalmente, o $\mathrm{N}$ é considerado fator limitante para o desenvolvimento do algodoeiro (Gossypium hirsutum var. latifolium Hutch) tanto em áreas irrigadas como no cultivo de sequeiro. Em doses adequadas estimula o crescimento e o florescimento, regulariza o ciclo da planta, aumenta a produtividade e melhora o comprimento e a resistência da fibra (Beltrão, 1999). Entretanto, aplicações excessivas podem causar crescimento vegetativo exagerado da planta e reduzir a produção e a qualidade da fibra (Hutmacher et al., 2004). O manejo eficiente da adubação nitrogenada é essencial para obtenção de altas produtividades, redução de custo, proteção ambiental e viabilização dos sistemas de produção existentes no Cerrado. O uso racional de corretivos e fertilizantes depende da disponibilidade de nutrientes no solo, da exigência nutricional da cultura, dos fatores que afetam sua eficiência, das relações econômicas entre os preços da pluma e dos insumos, além do impacto sobre o meio ambiente.

Klotz et al. (2003) afirmaram que o crescimento da adoção de práticas de agricultura de precisão e o monitoramento ambiental requerem novos sistemas e técnicas de aquisição de dados visando à informação detalhada do status da vegetação. Neste sentido, Kuckenberg et al. (2009) relataram que medidas de refletância permitem estimativas rápidas e não invasivas do estado nutricional das plantas (referentes ao N), auxiliando a adubação de precisão.

Sensores óticos acoplados em tratores que se deslocam pela lavoura podem coletar dados para monitorar o manejo da adubação nitrogenada e são menos dependentes das condições climáticas do que dados orbitais ou suborbitais (Steven, 2004). A eficiência dos sensores óticos é objeto de estudo de vários pesquisadores em diferentes culturas, como os realizados na cultura do algodão (Fridgen \& Varco, 2004; Sui et al., 2005; Motomiya et al., 2009; Zhao et al., 2005) além da cana-de-açúcar (Molin et al., 2010) e trigo (Povh et al., 2008; Grohs et al., 2009).

A eficácia de diferentes tratamentos desfolhantes do algodoeiro usando imagens aéreas multiespectrais, foi avaliada com sucesso por Yang et al. (2003). Zhao et al. (2005) observaram, avaliando o efeito de adubação nitrogenada e a aplicação de regulador de crescimento (PIX) no algodoeiro, que o baixo suprimento de $\mathrm{N}$ resultou em baixos níveis de clorofila na folha, estresse que foi determinado precocemente nas plantas com o aumento da refletância na faixa do visível, nos comprimentos de onda de 550 a $700 \mathrm{~nm}$.

Segundo Fridgen \& Varco (2004) a aplicação de diferentes doses de $\mathrm{N}$ induziu a uma ampla variação na concentração de $\mathrm{N}$ foliar e na de clorofila, de forma que a distinção de plantas deficientes em $\mathrm{N}$ pode ser detectada pela refletância foliar na faixa do visível ao infravermelho próximo, desde que nenhum outro nutriente esteja em falta na cultura. Por participar diretamente na formação da molécula existe uma forte relação entre nitrogênio e clorofila. Assim, conforme relataram Bullock \& Anderson (1998) com o aumento da disponibilidade de nitrogênio para a planta mais clorofila é produzida nas folhas, melhorando a percepção da cor verde da folha. Este aumento da clorofila, porém, atinge um patamar designado como ponto de maturidade fotossintética a partir do qual se mantém invariável, mesmo com o aumento da concentração de nitrogênio no tecido, o que explica o comportamento quadrático dos índices espectrais.

Sensores óticos dedicados permitem o rápido diagnóstico de condições de estresse das plantas, como aquelas causadas pela deficiência de N. Assim, este trabalho teve por objetivo avaliar a resposta espectral do dossel de plantas à variação de doses de nitrogênio $(\mathrm{N})$ e sua relação com os teores foliares de $\mathrm{N}$, de clorofila e produtividade, na cultura do algodoeiro.

\section{Material e MÉTODOS}

O experimento foi implantado em uma fazenda localizada no município de Chapadão do Sul, MS, cujas coordenadas geográficas são $18^{\circ} 46^{\prime} \mathrm{S}$ e $52^{\circ} 38^{\prime} \mathrm{O}$, em solo cultivado sob sistema de semeadura direta sobre palhada de inverno formada por milheto/sorgo e safrinha de milho, no delineamento em blocos casualizados, com quatro repetições. Os tratamentos consistiram de cinco doses de N: 0, 30, 70, 110 e $150 \mathrm{~kg} \mathrm{ha}^{-1}$, divididas em duas aplicações aos 28 e 41 dias após a emergência (DAE) utilizando-se, como fonte, o fertilizante ureia. Cada parcela foi constituída de quatro fileiras de semeadura com 7,0 $\mathrm{m}$ de comprimento espaçadas $0,9 \mathrm{~m}$ entre si; como área útil foram consideradas as duas fileiras centrais, eliminando-se 0,5 m de cada extremidade.

O solo da área experimental apresentou as seguintes características químicas na camada de $0-20 \mathrm{~cm}: \mathrm{pH}\left(\mathrm{CaCl}_{2}\right.$ 0,01 $\left.\mathrm{mol} \mathrm{L}^{-1}\right)$ 5,5; 30,1 $\mathrm{g} \mathrm{dm}^{-3}$ de MO; 13,6 mg dm${ }^{-3}$ de P; 3,7 mmolc dm ${ }^{-3}$ de $\mathrm{K} ; 4,3$ mmolc dm ${ }^{-3} \mathrm{de} \mathrm{Ca} ; 1,7$ mmolc dm$^{-3} \mathrm{de} \mathrm{Mg}$; saturação por bases (V) de $65 \%$. A semeadura do algodoeiro foi realizada mecanicamente no dia 11/12/2008 e a adubação de semeadura, com base na análise do solo. Os tratamentos fitossanitários e os demais tratos culturais foram realizados conforme descrito por EMBRAPA (2001) para garantir o bom desenvolvimento das plantas de maneira uniforme, em todos os tratamentos.

Os dados espectrais foram coletados com um sensor ótico ativo (Crop Circle ${ }^{\circledR}$ ACS-210, Holland Scientific, Inc.. Lincoln, NE, EUA) o qual emite a luz no comprimento de onda do vermelho a $580 \mathrm{~nm}$ (VIS) e do infravermelho próximo a $880 \mathrm{~nm}$ (IVP); a luz refletida é captada pelo detector; a partir da refletância ele calcula o índice de vegetação por diferença normalizada (NDVI). De acordo com o fabricante, a altura de trabalho do sensor pode variar de 0,25 a 2,15 m de distância ao alvo sendo que as leituras foram realizadas a uma altura padronizada de $0,60 \mathrm{~cm}$ das plantas e as leituras dos componentes do NDVI foram realizadas aos 28, 35, 41, 56, 64 e 91 dias após a emergência das plantas.

Realizaram-se leituras com o medidor portátil de clorofila ClorofiLOG CFL1030 (Falker Automação, Porto Alegre, RS) o qual consiste de um sensor com habilidade para medir o índice de clorofila (ou "índice de verde") presente na folha a partir da absorbância de energia nos comprimentos de onda de 650 e $940 \mathrm{~nm}$; este aparelho fornece as leituras dos índices de clorofila a, b e total; é operado manualmente e as leituras são localizadas 
em áreas de aproximadamente 2 x $3 \mathrm{~mm}$ sobre a folha. As leituras com o clorofilômetro foram realizadas na quinta folha completamente expandida a partir do ápice das plantas, em cinco plantas por parcela, nos mesmos dias das leituras com o sensor óptico.

A amostragem de tecido foliar foi realizada aos 91 DAE, no estádio de florescimento pleno, através de uma amostra composta de 10 folhas por parcela, para determinação dos teores de macronutrientes, conforme métodos descritos por Malavolta et al. (1997). A colheita manual do experimento ocorreu em 15/07/2009.

O efeito dos tratamentos e da relação entre as variáveis foi avaliado por meio de análise de variância, verificando-se a significância pelo teste $\mathrm{F}$ de Snedecor, análise de regressão e correlação linear de Pearson.

\section{RESULTADOS E DISCUSSÃO}

As doses de $\mathrm{N}$ aplicadas ocasionaram variação significativa nos teores foliares de $\mathrm{N}$. No tratamento em que o $\mathrm{N}$ não foi aplicado, os teores foliares estiveram abaixo da faixa de suficiência preconizada por Silva (1999) visto que variam entre 35 a $43 \mathrm{~g} \mathrm{~kg}^{-1}$. A análise de regressão revelou ajuste quadrático entre doses e teores foliares de N (Figura 1) cujos resultados são semelhantes aos encontrados por Brandão et al. (2009) e Motomiya et al. (2009).

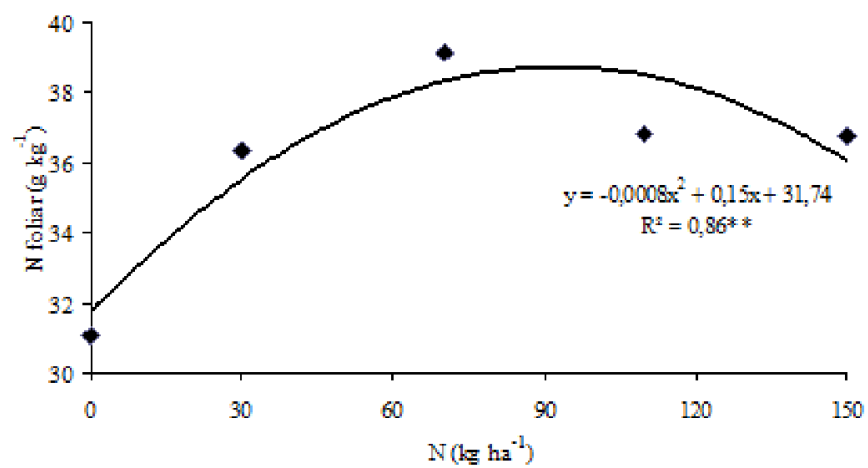

Figura 1. Relação entre doses de $\mathrm{N}$ aplicadas e teores foliares de N, no algodoeiro

Os tratamentos com $\mathrm{N}$ resultaram em amplas variações nas refletâncias no IVP, visível e no NDVI. Os valores do NDVI, conforme descrito por Rouse et al. (1973) variam de +1 a -1 e aumentam com o aumento da diferença das refletâncias no visível e no infravermelho. Na Tabela 1 se encontram a média e o coeficiente de variação dos dados nos seis períodos de avaliação. Embora o NDVI tenha aumentado, os valores do IVP e do visível (VIS) apresentaram, inicialmente, um aumento para depois diminuir, também observado por Motomiya et al. (2009) e Grohs et al. (2009). Os coeficientes de variação foram baixos para o NDVI enquanto para o IVP e visível houve aumento do CV em estádios mais avançados de crescimento da cultura indicando que essas variáveis isoladas podem ser menos confiáveis na identificação de deficiências nutricionais na cultura em estádio avançado de crescimento.
Tabela 1. Valores da média e coeficientes de variação para as leituras das refletâncias no comprimento de onda no vermelho a $580 \mathrm{~nm}$ (IVP) e infravermelho a $880 \mathrm{~nm}$ (VIS) e do índice de vegetação por diferença normalizada (NDVI) em função do período de coleta de dados (dias após a emergência - DAE)

\begin{tabular}{|c|c|c|c|}
\hline & IVP & VIS & NDVI \\
\hline $\begin{array}{l}\text { Média } \\
\text { CV }\end{array}$ & $\begin{array}{l}0,80 \\
9,65\end{array}$ & $\begin{array}{r}28 \mathrm{DAE}^{1} \\
0,26 \\
6,77\end{array}$ & $\begin{array}{l}0,50 \\
6,09\end{array}$ \\
\hline $\begin{array}{l}\text { Média } \\
\text { CV }\end{array}$ & $\begin{array}{r}1,79 \\
13,29\end{array}$ & $\begin{array}{r}35 \text { DAE } \\
0,40 \\
4,80\end{array}$ & $\begin{array}{l}0,61 \\
5,86\end{array}$ \\
\hline $\begin{array}{l}\text { Média } \\
\text { CV }\end{array}$ & $\begin{array}{r}0,95 \\
16,61 \\
\end{array}$ & $\begin{array}{r}1 \mathrm{DAE}^{2} \\
0,20 \\
8,60\end{array}$ & $\begin{array}{l}0,62 \\
7,64\end{array}$ \\
\hline $\begin{array}{l}\text { Média } \\
\text { CV }\end{array}$ & $\begin{array}{l}0,90 \\
9,66 \\
\end{array}$ & $\begin{array}{r}56 \text { DAE } \\
0,11 \\
4,88 \\
\end{array}$ & $\begin{array}{l}0,77 \\
3,47 \\
\end{array}$ \\
\hline $\begin{array}{l}\text { Média } \\
\text { CV }\end{array}$ & $\begin{array}{r}0,81 \\
19,91\end{array}$ & $\begin{array}{r}64 \text { DAE } \\
0,11 \\
9,20\end{array}$ & $\begin{array}{l}0,75 \\
7,30\end{array}$ \\
\hline $\begin{array}{l}\text { Média } \\
\text { CV }\end{array}$ & $\begin{array}{r}2,83 \\
23,32\end{array}$ & $\begin{array}{r}91 \mathrm{DAE}^{3} \\
0,52 \\
66,67\end{array}$ & $\begin{array}{l}0,78 \\
3,52\end{array}$ \\
\hline
\end{tabular}

$28 \mathrm{DAE}^{1}$ - primeira aplicação de ureia em cobertura; $41 \mathrm{DAE}^{2}$ - segunda aplicação de ureia em cobertura; $91 \mathrm{DAE}^{3}$ - amostragem de tecido foliar

Em geral, os valores do NDVI aumentaram com o aumento das doses de $\mathrm{N}$ e com o estádio fenológico do algodoeiro (Figura 2). Nas fases iniciais de crescimento a cobertura reduzida do solo pela cultura, devido ao baixo índice de área foliar, ocasionou menores valores de NDVI. Conforme a planta se desenvolve, ocorre um aumento da cobertura vegetal reduzindo a influência do solo na resposta espectral. Esses resultados são condizentes com as afirmações de Epiphanio et al. (1996) ao observarem que, a medida em que a cultura se desenvolve a média dos índices de vegetação aumenta e a variância diminui fazendo com que o coeficiente de variação seja cada vez menor.

Por outro lado, a refletância na região do infravermelho(IVP) apresentou um pequeno incremento entre a primeira e a segunda leituras, voltando a apresentar redução até os 64 DAE; aos 91 DAE, entretanto, elevou-se, atingindo uma média de 2,83, quando a cultura apresentava seu máximo crescimento vegetativo. Com o aumento do índice de área foliar houve aumento da refletância na região do infravermelho. Nesta região, a partir de $760 \mathrm{~nm}$ a radiação incidente sobre a vegetação é fortemente espalhada, sofrendo múltiplas reflexões e transmissões, de tal forma que, quanto maior for a biomassa maior também será o espalhamento e, em consequência, a energia refletida (Amaral et al., 1990).

As relações entre doses de $\mathrm{N}$ aplicado e o NDVI se ajustaram ao modelo quadrático nas avaliações feitas aos 35, 41 e 56 DAE (Figura 2A). Referente à refletância no IVP, constatou-se efeito significativo apenas aos 35 e 41 DAE (Figura 2B). Souza et al. (2009) observaram aumento do NDVI e refletância no IVP com o aumento da taxa de $\mathrm{N}$ aplicada na cultura do milho.

A relação entre doses de $\mathrm{N}$ aplicado e a refletância no visível apresentou diferenças significativas aos 64 DAE ajustando-se 

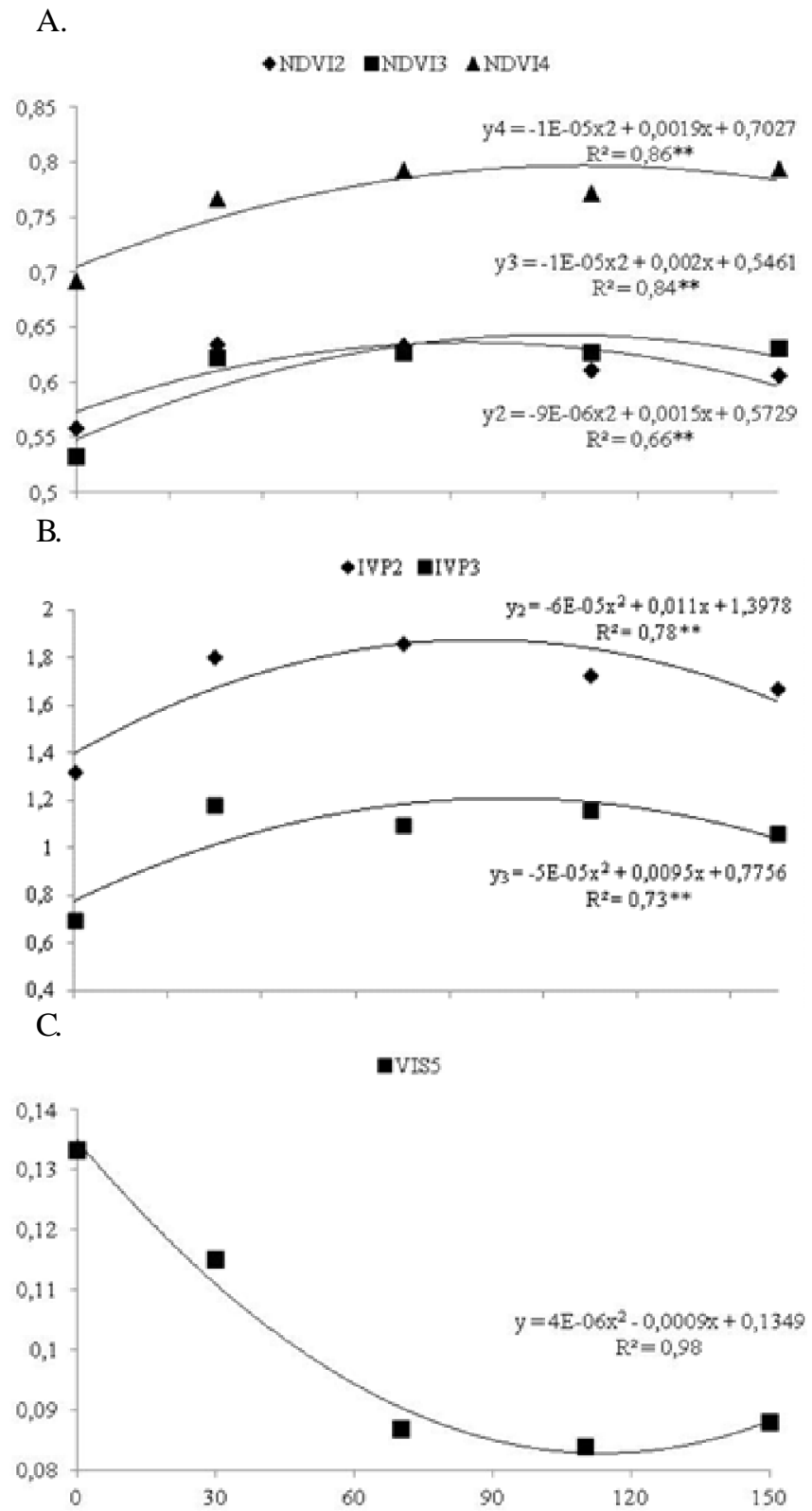

Figura 2. Relação entre doses de $\mathrm{N}$ aplicado e NDVI, no algodoeiro, aos 35 (NDVI2), 41 (NDVI3) e 56 DAE (NDVI4) (A); relação entre doses de $N$ aplicado e a refletância no infravermelho próximo no al godoeiro, aos 35 (IVP2) e 41 DAE (IVP3) (B); relação entre doses de N aplicado e a refletância no visível aos 64 D AE (VIS5) (C)

ao modelo quadrático (Figura 2C) sendo que a refletância foi maior nas doses mais baixas. Peñuelas et al. (1994) também observaram que plantas de girassol com deficiência de $\mathrm{N}$ apresentaram maiores refletâncias no visível e menores no IVP. Os autores ressaltaram que o envelhecimento das folhas pode ser identificado pelo aumento da refletância no visível. As variações na refletância espectral de plantas com deficiência de $\mathrm{N}$ podem ser resultado de mudanças na estrutura $\mathrm{e}$ composição foliar, o que inclui a concentração de pigmentos e o tamanho das células.

Com a evolução das fases fenológicas da cultura do algodoeiro, os teores foliares de clorofila a, b e total aumentaram (Figura 3). As leituras intermediárias (2, 3, 4 e 5) foram
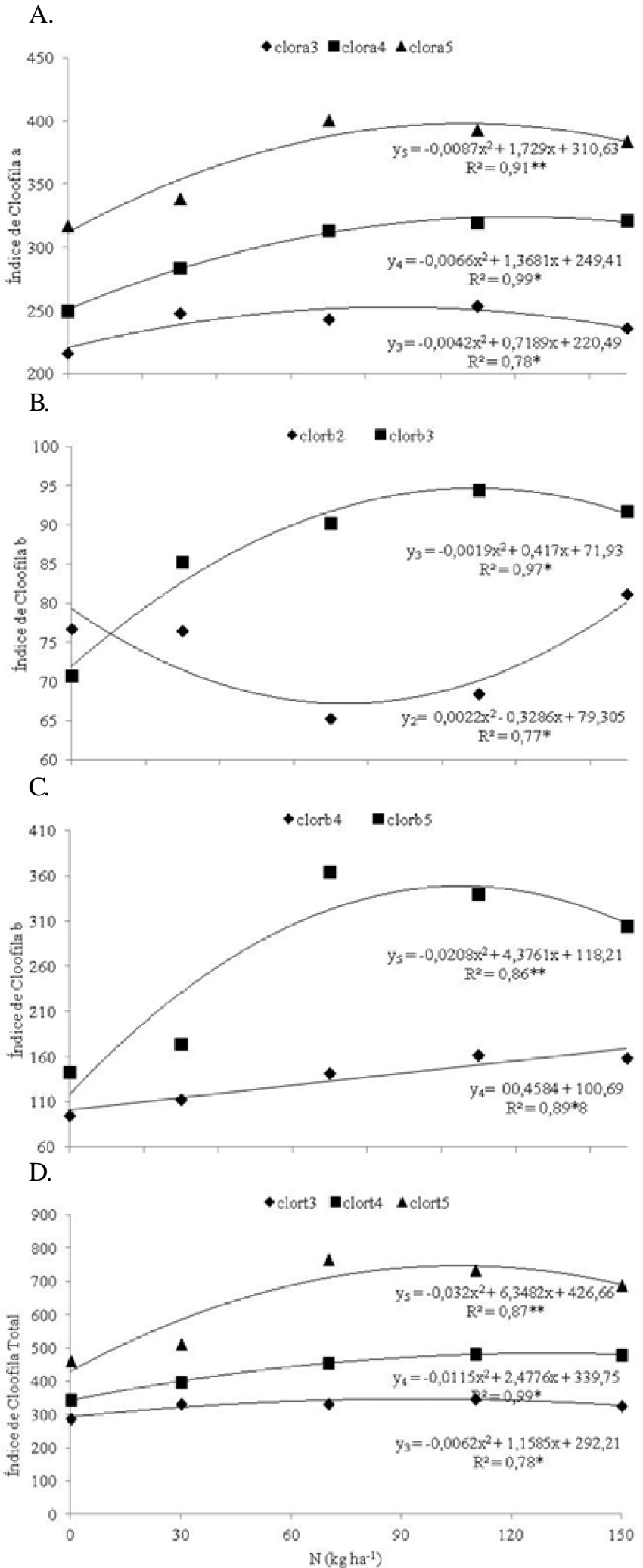

Figura 3. Relação entre doses de $\mathrm{N}$ aplicado e índice de clorofila a, no algodoeiro, aos 41 (clora3), 56 (clora4) e 64 DAE (clora5) (A); relação entre doses de $\mathrm{N}$ aplicado e índice de clorofila b, aos 35 (clorb2, 41 (clorb3) (B), relação entre doses de $\mathrm{N}$ aplicado e índice de clorofila $\mathrm{b}$, aos 56 (clorb4) e 64 DAE (clorb5) (C); relação entre doses de $\mathrm{N}$ aplicado e índice de clorofila total, no algodoeiro, aos 41 (clort3), 56 (clort4) e 64 D AE (clort5) (D) 
relacionadas significativamente com as doses de $\mathrm{N}$ aplicadas apresentando respostas quadráticas e altamente significativas.

Com a deficiência de $\mathrm{N}$ ocorre diminuição da concentração de clorofila, do conteúdo de proteína solúvel, da taxa de expansão foliar e do desenvolvimento da planta (Read et al., 2002). Como a refletância na região do visível varia em função da concentração de clorofila no tecido foliar, quanto menor o suprimento de $\mathrm{N}$ na planta, menor também será o índice de clorofila e, em contrapartida, menor será a absorção da radiação na região do visível, o que causa alterações no NDVI. Motomiya et al. (2009) observaram diferenças mais acentuadas entre a ausência de adubação nitrogenada e doses variando de 50 a $200 \mathrm{~kg}^{-1}$, não havendo grande distinção entre essas doses, ao longo de um período de coleta de dados.

A produtividade do algodoeiro aumentou significativamente com as doses aplicadas (Figura 4) ajustando-se ao modelo quadrático. As doses utilizadas não permitiram alcançar o ponto de máxima produtividade o qual, conforme a derivação da equação de regressão, seria atingido apenas na dose de 195 $\mathrm{kg} \mathrm{ha}^{-1}$.

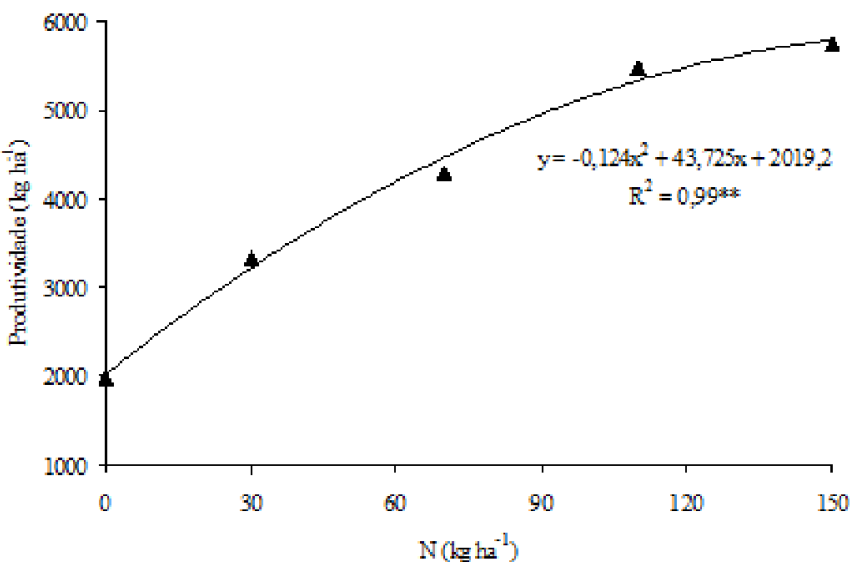

Figura 4. Curva de regressão ajustada para a produtividade do al go doeiro em função das doses de $\mathrm{N}$ aplicadas

As doses de $\mathrm{N}$ aplicadas ao solo apresentaram ajuste quadrático com a altura das plantas (Figura 5). Como o $\mathrm{N}$ é constituinte de muitos componentes da célula vegetal, como aminoácidos e ácidos nucleicos, a deficiência rapidamente inibe

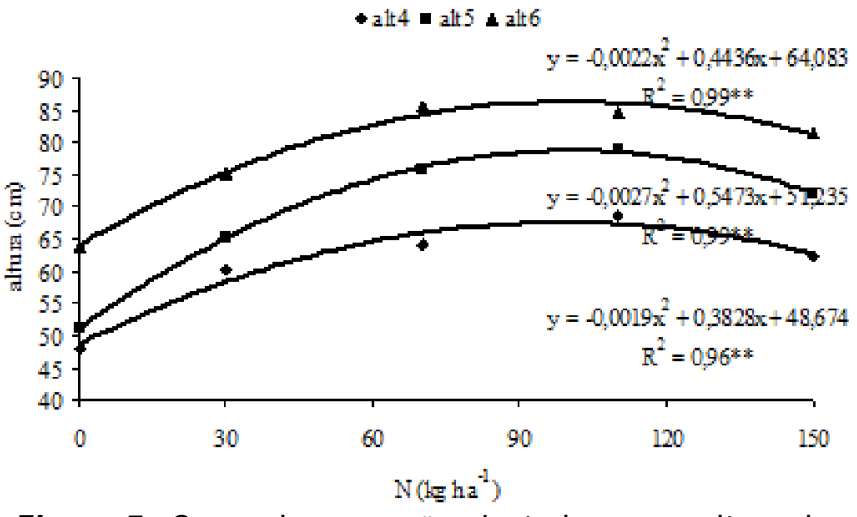

Figura 5. Curva de regressão ajustada para a altura do algodoeiro em função das doses de $\mathrm{N}$ aplicadas aos 56 (alt4), 64 (alt5) e 91 D AE (alt6) o crescimento vegetal e, caso esta deficiência persista, podem ocorrer clorose e abscisão de folhas; se a deficiência se desenvolve lentamente os caules se tornam pronunciadamente delgados e, comumente, lenhosos, devido ao acúmulo excessivo de carboidratos que não podem ser utilizados na síntese de aminoácidos e outros compostos nitrogenados (Taiz \& Zeiger, 2004).

Somente a partir de 56 DAE (Tabela 2) a produtividade foi correlacionada significativamente com o NDVI, com diminuição gradativa da correlação até a última leitura, aos 91 DAE; é provável que este evento tenha ocorrido em virtude das folhas com baixos teores de $\mathrm{N}$ apresentarem menores teores de clorofila e, consequentemente, maior refletância e transmitância no visível (400-700 nm) e baixas refletância e transmitância no infravermelho próximo (Noh et al., 2005) o que ocasiona alterações nos valores de NDVI e VIS/IVP.

Tabela 2. Correlações entre o índice de vegetação por diferença normalizada (NDVI), índices de clorofila e altura de plantas com a produtividade do algodoeiro

\begin{tabular}{|c|c|c|c|c|c|c|}
\hline & \multicolumn{6}{|c|}{ Dias após emergência (DAE) } \\
\hline & 28 & 35 & 41 & 56 & 64 & 91 \\
\hline & \multicolumn{6}{|c|}{ NDVI } \\
\hline \multirow{2}{*}{ Prod. } & $0,25^{\text {ns }}$ & $0,18^{\text {ns }}$ & $0,33^{\text {ns }}$ & $0,72 * *$ & $0,61 * *$ & $0,52^{*}$ \\
\hline & \multicolumn{6}{|c|}{ Clorofila a } \\
\hline \multirow[t]{2}{*}{ Prod. } & $-0,25^{\mathrm{ns}}$ & $-0,24^{n s}$ & $0,36^{\text {ns }}$ & $0,79 * *$ & $0,75^{* *}$ & $0,26^{\mathrm{ns}}$ \\
\hline & \multicolumn{6}{|c|}{ Clorofila b } \\
\hline \multirow[t]{2}{*}{ Prod. } & $-0,09^{n s}$ & $-0,13^{\mathrm{ns}}$ & $0,52 *$ & $0,83 * *$ & $0,77 * *$ & $0,20^{\text {ns }}$ \\
\hline & \multicolumn{6}{|c|}{ Clorofila total } \\
\hline Prod. & $-0,18^{\text {ns }}$ & $-0,21^{\mathrm{ns}}$ & $0,44^{*}$ & $0,82 * *$ & $0,77 * *$ & $0,23^{\mathrm{ns}}$ \\
\hline & \multicolumn{6}{|c|}{ Altura } \\
\hline Prod. & $-0,20^{\mathrm{ns}}$ & $0,36^{\mathrm{ns}}$ & $0,52^{*}$ & $0,84 * *$ & $0,88^{* *}$ & $0,78 * *$ \\
\hline
\end{tabular}

A maior correlação entre os índices de clorofila com a produtividade também foi observada aos 56 DAE, embora o índice de clorofila b e o total tenham apresentado correlações significativas com a produtividade aos 41 DAE, uma vez que neste período ainda é possível fazer suplementação da adubação. Entre as duas clorofilas a produtividade foi mais fortemente correlacionada com a clorofila b do que com a clorofila a. Segundo Victório et al. (2007) a clorofila a é a responsável pela eficiência fotossintética enquanto a clorofila b e os carotenoides são os pigmentos acessórios ativos na obtenção de energia. Jinwen et al. (2009) observaram, na cultura do arroz, que as leituras com o clorofilômetro SPAD (Konica Minolta Sensing, Inc., Sakai, Osaka, Japão) se tornaram mais e mais sensíveis às taxas de $\mathrm{N}$, conforme a idade da planta tenha aumentado.

Correlações altamente significativas foram observadas entre o NDVI e o índice de clorofila total (Tabela 3) a partir da terceira leitura (41 DAE) até a quinta leitura (64 DAE); aos 91 DAE não houve correlação significativa entre o teor de clorofila total e o NDVI; tais resultados estão de acordo com as afirmações de Fridgen \& Varco (2004) os quais consideraram que a distinção de plantas deficientes em nitrogênio pode ser detectada pela refletância foliar na faixa do visível ao infravermelho próximo, desde que não haja outras deficiências na cultura. 
Tabela 3. Correlações entre o índice de vegetação por diferença normalizada (N DVI) e o índice de clorofila total na cultura do al godoeiro

\begin{tabular}{ccccccc}
\hline NDVI & \multicolumn{6}{c}{ Dias após emergência (DAE) } \\
\cline { 2 - 7 } (DAE) & $\mathbf{2 8}$ & $\mathbf{3 5}$ & $\mathbf{4 1}$ & $\mathbf{5 6}$ & $\mathbf{6 4}$ & $\mathbf{9 1}$ \\
28 & $-0,17^{\text {ns }}$ & $-0,02^{\text {ns }}$ & $0,43^{\text {ns }}$ & $-0,01^{\text {ns }}$ & $0,23^{\text {ns }}$ & $0,09^{\text {ns }}$ \\
35 & $0,03^{\text {ns }}$ & $0,08^{\text {ns }}$ & $0,43^{\text {ns }}$ & $0,00^{\text {ns }}$ & $0,13^{\text {ns }}$ & $-0,13^{\text {ns }}$ \\
41 & $0,03^{\text {ns }}$ & $0,13^{\text {ns }}$ & $0,61^{* *}$ & $0,11^{\text {ns }}$ & $0,23^{\text {ns }}$ & $0,00^{\text {ns }}$ \\
56 & $-0,12^{\text {ns }}$ & $-0,04^{\text {ns }}$ & $0,70^{* *}$ & $0,60^{* *}$ & $0,59^{* *}$ & $-0,01^{\text {ns }}$ \\
64 & $-0,06^{\text {ns }}$ & $-0,11^{\text {ns }}$ & $0,09^{\text {ns }}$ & $0,77^{* *}$ & $0,67 * *$ & $0,27^{\text {ns }}$ \\
91 & $-0,13^{\text {ns }}$ & $-0,23^{\text {ns }}$ & $0,42^{\text {ns }}$ & $0,41^{\text {ns }}$ & $0,33^{\text {ns }}$ & $-0,14^{\text {ns }}$ \\
\hline
\end{tabular}

DAE: dias após a emergência. ${ }^{* *}$ significativo a $1 \%$ de probabilidade

Os resultados obtidos neste trabalho indicam que o sensor se torna mais sensível à concentração foliar de $\mathrm{N}$ com a evolução das fases fenológicas da cultura, exceto quando esta atinge alto índice de área foliar (IAF) e começa a entrar em senescência. Em geral, o NDVI atinge a saturação em condições de média a alta quantidade de biomassa (Gitelson et al., 2002). Segundo Moreira (2001) o NDVI aumenta linearmente com o aumento do IAF e então assume uma forma assintótica a partir de onde o NDVI aumenta pouco, relativamente ao aumento do IAF. A diminuição da sensibilidade do NDVI em altos valores de IAF ocorre devido a refletância ser atenuada quando a superfície do solo está completamente encoberta pelas folhas. Esta saturação ocorre em razão da quantidade de luz vermelha que pode ser absorvida pelas folhas, alcançando rapidamente um pico; em contraste, a radiação no IVP difundida pelas folhas continua a aumentar quando o IAF excede 3,0 (Gitelson et al., 2002). Como resultado, quando o dossel atinge $100 \%$ a refletância no IVP continua a aumentar mas a refletância no vermelho apresentará pequena diminuição resultando em mudanças suaves na razão. Embora esta característica do NDVI seja uma deficiência para algumas aplicações, tais como inferência da biomassa total, pode também ser uma vantagem para identificar os valores de NDVI nos quais as superfícies estão completamente cobertas por vegetação. Povh et al. (2008) observaram que o aumento de biomassa com o aumento das doses de N foi suficiente para saturar o NDVI, desde a primeira leitura na cultura do milho utilizando sensor que emite radiação ativa em comprimentos de onda centralizados no vermelho (660 $\mathrm{nm})$ e no infravermelho próximo $(770 \mathrm{~nm})$.

\section{Conclusões}

1. A resposta espectral e o índice de clorofila das plantas do algodoeiro foram influenciados pelas doses de $\mathrm{N}$ aplicadas.

2. O NDVI obtido através de sensor óptico ao nível terrestre permite estimar, indiretamente, os teores foliares de N, o índice de clorofila e a produtividade em plantas de algodoeiro.

\section{LiTERATURA CITADA}

Amaral, S.; Kazmierczark, M. L.; Zerbiani, N. J.; Carvalho, V. C. Comportamento espectral de mudas de Eucalyptus grandis (Hill) Maiden em diferentes percentagens de cobertura. São José dos Campos: INPE, 1990. 13p. Documentos INPE-5247PRE/1678
Beltrão, N. E. de M. Algodão brasileiro em relação ao mundo: Situação e perspectivas. In: Beltrão, N. E. de M. (ed) O agrone-gócio do algodão no Brasil. Brasília: Embrapa Comunicação para Transferência de Tecnologia, 1999, v.1, p.15-27.

Brandão, Z. N.; Sofiatti, V.; Ferreira, G. B.; Lima, R. L. S.; Beltrão, N. E. de M.; Silva, B. B. da. Predição da adubação nitrogenada através da utilização do índice spad para o algodoeiro no semiárido. Engenharia Ambiental, v.6, p.368382, 2009.

Bullock, D. G.; Anderson, D. S. Evaluation of the Minolta SPAD 502 chlorophyll meter for nitrogen management in corn. Journal of Plant Nutrition, v.21, p.741-755, 1998.

EMBRAPA - Empresa Brasileira de Pesquisa Agropecuária. Algodão: Tecnologia de produção. Dourados: Embrapa Agropecuária Oeste, 2001.296p.

Epiphanio, J. C. N.; Gleriani, J. M.; Formaggio, A. R.; Rudorff, B. F. T. Índices de vegetação na cultura do feijão. Pesquisa Agropecuária Brasileira, v.31, p.445-454, 1996.

Fridgen, J. L.; Varco, J. J. Dependency of cotton leaf nitrogen, chlorophyll, and reflectance on nitrogen and potassium availability. Agronomy Journal, v.96, p.63-69, 2004.

Gitelson, A. A.; Stark, R.; Grits, U.; Rundquist, D.; Kaufman, Y.; Derry, D. Vegetation and soil lines in visible spectral space: A concept and technique for remote estimation of vegetation fraction. International Journal of Remote Sensing, v.23, p.2537-2562, 2002.

Grohs, D. S.; Bredemeier, C.; Mundstock, C. M.; Poletto, N. Modelo para estimativa do potencial produtivo em trigo e cevada por meio do sensor GreenSeeker. Engenharia Agrícola, v.29, p.101-112, 2009.

Hutmacher, R. B.; Travis, R. L.; Rains, D. W.; Vargas, R. N.; Roberts, B. A.; Weir, B. L.; Wright, S. D.; Munk, D. S.; Marsh, B. H.; Keeley, M. P.; Fritschi, F. B.; Munier, D. J.; Nichols, R. L.; Delgado. R. Response of recent Acala cotton cultivars to variable nitrogen rates in the San Joaquin valley of California. Agronomy Journal, v.96, p.48-62, 2004.

Jinwen L.; Jingping, Y.; Pinpin, F.; Junlan, S.; Dongsheng, L.; Changshui, G.; Wenyue, C. Responses of rice leaf thickness, SPAD readings and chlorophyll $\mathrm{a} / \mathrm{b}$ ratios to different nitrogen supply rates in paddy field. Field Crops Research, v.114, p.426-432, 2009.

Klotz, P.; Bach, H.; Mauser, W. GVIS - Ground-operated visible/ near infrared imaging spectrometer. In: Stafford, J.; Werner, A. (ed) European Conference on Precision Agriculture, 3, 2003, Montpellier. Proceedings... Montpellier: AgroMontpellier, 2003. p.35-39.

Kuckenberg, J.; Tartachnyk, I.; Noga, G. Detection and differentiation of nitrogen-deficiency, powdery mildew and leaf rust at wheat leaf and canopy level by laser-induced chlorophyll ûuorescence. Biosystems Engineering, v.103, p.121-128, 2009.

Malavolta, E.; Vitti, G. C.; Oliveira, S. A. Avaliação do estado nutricional das plantas: Princípios e aplicações. 2.ed. Piracicaba: Potafos, 1997. 201p.

Molin, J. P; Frasson, F. R.; Amaral, L. R.; Povh, F. P.; Salvi, J. V. Capacidade de um sensor ótico em quantificar a resposta da cana-de-açúcar a doses de nitrogênio. Revista Brasileira de Engenharia Agrícola e Ambiental, v.14, p.1345-1349, 2010. 
Moreira, M. A. Fundamentos do sensoriamento remoto e metodologias de aplicação. São José dos Campos: Instituto Nacional de Pesquisa Espacial, 2001. 205p.

Motomiya, A. V. A., Molin, J. P.; Chiavegato, E. D. Utilização de sensor óptico ativo para detectar deficiência foliar de nitrogênio em algodoeiro. Revista Brasileira de Engenharia Agrícola e Ambiental, v.13, p.137-145, 2009.

Noh, H.; Zhang, Q.; Han, S.; Shin, B.; Reum, D. Dynamic calibration and image segmentation methods for multispectral imaging crop nitrogen deficiency sensors. Transactions of the ASAE, v.48, p.393-401, 2005.

Peñuelas, J.; Gamon, J. A.; Fredeen, A. L.; Merino, J.; Field, C. B. Reflectance indices associated with physiological changes in nitrogen and water-limited sunflower leaves. Remote Sensing Environment, v.48, p.135-146, 1994.

Povh, F. P.; Molin, J. P.; Gimenez, L. M.; Pauletti, V.; Molin, R.; Salvi, J. V. Comportamento do NDVI obtido por sensor ótico ativo em cereais. Pesquisa Agropecuária Brasileira, v.23, p.1075-1083, 2008.

Read, J. J.; Tarpley, L.; Mckinion, J. M.; Reddy, K. R. Narrowwaveband reflectance for remote estimation of nitrogen status in cotton. Journal of Environmental Quality, v.31, p.1442-1452, 2002.

Rouse, J. W.; Haas, R. H.; Schell, J. A.; Deering, D. W. Monitoring vegetation systems in the great plains with ERTS. In: ERTS Symposium, 3, Washignton. Proceedings..., Washignton: NASA, v.1, 1973. p.309-317.
Silva, N. M. Nutrição mineral e adubação do algodoeiro no Brasil. In: Cia, E.; Freire, E. C.; Santos, W. J. (ed.). Cultura do algodoeiro. Piracicaba: Potafos, 1999. p.57-92.

Souza, E. G.; Rocha, T.; Uribe-Opazo, M. A.; Nóbrega, L. H. P. Índices de vegetação no milho em função da hora do dia e da taxa de nitrogênio aplicada. Revista Brasileira de Engenharia Agrícola e Ambiental, v.13, p.865-872, 2009.

Steven, M. D. Correctong the effects of field of view and varying illumination in spectral measurementes of crops. Precision Agriculture, v.5, p.55-72, 2004.

Sui, R.; Wilkerson, J. B.; Hart, W. E.; Wilhelm, L. R., Howard, D. D. Multi-spectral sensor for detection of nitrogen status in cotton. Applied Engineering in Agriculture, v.21, p.167-172, 2005.

Taiz, L.; Zeiger, E. Fisiologia vegetal. 3.ed. Porto Alegre: Artmed, 2004. 720p.

Victório, C. P.; Kuster, R. M.; Lage, C. L. S. Qualidade de luz e produção de pigmentos fotossintéticos em plantas in vitro de Phyllanthus tenellus roxb. Revista Brasileira de Biociências, v.5, p.213-215, 2007.

Yang, C.; Greenberg, S. M.; Everitt, J. H.; Sappington, T. W.; Norman Jr., J. W.; Evaluation of cotton defoliation strategies using airborne multispectral imagery. Transactions of the ASAE, v.46, p.869-876, 2003.

Zhao, D.; Reddy, R. K.; Kakani, V. G.; Read, J. J.; Koti, S. Selection of optimum reflectance ratios for estimating leaf nitrogen and chlorophyll concentrations of field-grown cotton. Agronomy Journal, v.97, p.89-98, 2005. 